\title{
Effect of the grounding system arrangement on the lightning current along tall structures
}

\begin{abstract}
The behaviour of lightning current along tall structures is an important case to be evaluated. The behaviour is influenced by a few factors of which one is the ground or earth reflection factor. Previous studies have assumed that the ground reflection factor is a constant value and this value is usually used when evaluating the lightning current along a tall tower. This study presents the behaviour of lightning current along a tower, including variation in the value of the ground reflection factor. The simulation work is done by selecting the sum of two Heidler functions as a current channel base and a distributed source representation current is used to simulate the lightning current along the tower and channel. The ground reflection factor is calculated based on the correlation between tower and ground impedances whereby the ground impedance is dependent on a circle grounding system arrangement. The results indicate that by increasing the length of the rods, the value of the ground impedance decreases, but the peak value of the current along the tower is more dependent on the value of the ground reflection factor. It is shown that by increasing the length of the rods, the current peaks increase. Thus, the outcome of this study may be of benefit to Electrical Engineers to set an appropriate scheme of protection for the equipment which is installed along the tower, based on the effect of the grounding system arrangement for different conditions of soil resistivity.
\end{abstract}

Keyword: Component; Ground reflection factor; Grounding system; Lightning; Tall structures; Telecommunications tower 